\title{
An automated methodology for performing time synchronous averaging of a gearbox signal without speed sensor
}

\author{
F. Combet*, L. Gelman \\ Applied Mathematics and Computing Group, School of Engineering, \\ Cranfield University, Cranfield MK43 OAL, UK
}

\begin{abstract}
In this paper we extend a sensorless algorithm proposed by Bonnardot \& al. for angular resampling of the acceleration signal of a gearbox submitted to limited speed fluctuation. The previous algorithm estimates the shaft angular position by narrowband demodulation of one harmonic of the mesh frequency. The harmonic was chosen by trial and error. This paper proposes a solution to select automatically the mesh harmonic used for the shaft angular position estimation. To do so it evaluates the local signal to noise ratio associated to the mesh harmonic and deduces the associated low-pass filtering effect on the time synchronous average (TSA) of the signal. Results are compared with the TSA obtained when using a tachometer on an industrial gearbox used for wastewater treatment. The proposed methodology requires only the knowledge of an approximate value of the running speed and the number of teeth of the gears. It forms an automated scheme which can prove useful for real time diagnostic applications based on TSA where speed measurement is not possible or not advisable due to difficult environmental conditions.
\end{abstract}

Key words: Time Synchronous Averaging without speed sensor, gearbox vibration signal, automated methodology, condition monitoring applications.

\section{Introduction}

Time domain averaging or time synchronous averaging (TSA) is a well adopted signal processing technique which enables periodic waveforms to be extracted from noisy signals $[1,2]$. It is particularly suited for the vibration analysis of

* f.combet@cranfield.ac.uk 
mechanical systems such as gearboxes, as it enables the vibration of a single gear to be separated from the vibration of the complete system. This technique requires the measurement of the "trigger" signal, i.e. a signal phase-locked with the angular position of one rotating shaft in the system. This speed measurement is typically delivered by a tachometer which delivers one pulse per revolution. An optical encoder (with multiple pulses per revolution) can also be used for a better angular resolution.

In some particular environments however, a speed sensor could be rather inconvenient - if not impossible - to use for condition monitoring purposes. Optical encoders need to be attached to the end of a shaft, which is possible for limited applications only. Optical "pick-up" sensors (tachometers) could be easier to manipulate, but in some environments they can cause problems, for example in high ambient light conditions or where they are subjected to contaminants like dust, oil or steam. In these conditions, a condition monitoring methodology that would operate without the need of any speed measurement would be of great interest in practice.

Recently, an original method has been proposed by Bonnardot \& al. [3] for angular resampling of the vibration signal of a gearbox without the need of a speed sensor, when the gearbox is submitted to limited speed fluctuation (i.e. under relatively steady load condition). The idea was to estimate the angular position of the shaft by narrow-band demodulation of one harmonic of the mesh frequency. The harmonic was chosen by trial and error. However, in order for this method to be applicable in a real time and industrial environment, different parameters in the method need to be adjusted in an automated and reliable fashion, i.e. without the need of any visual inspection of the frequency spectrum of the signal or of the estimated speed.

The objective of this paper, which can be viewed as a extension of this previous work [3], is to propose an automated methodology in order to obtain the time synchronous averaged signal for any particular shaft in a gearbox without speed sensor. In that purpose, different points in the methodology must be discussed: which stage in the gearbox and which mesh harmonic to select, how to quantify the accuracy of the estimated speed and of the TSA, and which interpolation method to use for resampling the signal.

In section 2 we first briefly recall the method proposed in [3] for estimation of the instantaneous shaft angular position and we show that the phase error of the estimation can be quantified by the local signal to noise ratio of the demodulated mesh harmonic. In section 3 we discuss the interpolation method to use for performing angular resampling and TSA, and we quantify the lowpass filtering effect on the TSA due to the phase error by what we call the "cut-off order" of the TSA. These results are then illustrated in section 4 on an industrial gearbox used in wastewater treatment. Finally, a complete 
methodology is proposed in section 5 for the selection of the mesh harmonic to demodulate and the estimation of the TSA of a particular shaft in the gearbox.

\section{Shaft speed estimation from a mesh harmonic}

\subsection{Recall of the method}

The model of the vibration signal for one stage of the gearbox used in [3] and first proposed in [4] is:

$$
x(t)=\sum_{k=1}^{K} X_{k}\left(1+a_{k}(t)\right) \cos \left(2 \pi k f_{m} t+\phi_{k}(t)+\Phi_{k}\right)+b(t)
$$

where $X_{k}$ is the amplitude of the $k$ th mesh harmonic, $f_{m}$ is the average mesh frequency for the stage considered, $a_{k}(t)$ and $\phi_{k}(t)$ are the amplitude and phase modulation functions of mesh harmonic $k$ and $\Phi_{k}$ is the initial phase of harmonic $k . b(t)$ represents the additive background noise. The phase modulation of mesh harmonic $k$ is assumed proportional to that of the mesh fundamental $\phi_{m}(t)$ :

$$
\phi_{k}(t)=k \phi_{m}(t)
$$

The mesh frequency $f_{m}$ is linked to the two rotation speeds $f_{1}$ and $f_{2}$ of the gears in mesh by:

$$
f_{m}=N_{1} f_{1}=N_{2} f_{2}
$$

where $N_{1}$ and $N_{2}$ are the corresponding number of teeth of the meshing gears. Figure 1 presents an example of a mesh frequency with its surrounding first order sidebands. Spectral components (mesh and sidebands) are smeared due to slight speed fluctuations. In order to extract the instantaneous speed, a narrow-band filter centred at the mesh frequency and with a bandwidth equal to $2 B$ is applied. The choice of $\mathrm{B}$ will be discussed later on part 2.2. The closest sidebands around the mesh frequency are those of the meshing gear with the lower rotation speed. Since these sidebands are modulated the same way as the mesh frequency due to speed fluctuations, the maximum frequency fluctuation of the $k$ th mesh harmonic $\delta f_{k}$ that causes no overlap is [3]:

$$
\delta f_{k} \leq B_{\max }
$$


with $B_{\max }$ the maximum width of the band-pass filter:

$$
B_{\text {max }}=\min \left(f_{1}, f_{2}\right) / 2=\frac{f_{m}}{2 \max \left(N_{1}, N_{2}\right)}
$$

This maximum bandwidth fixes the maximum fluctuation allowed by the method: since $f_{m}=f_{k} k$, the maximum allowed relative speed fluctuation for mesh harmonic $k$ is:

$$
\frac{\delta f_{k}}{f_{k}} \leq \frac{1}{2 k \max \left(N_{1}, N_{2}\right)}
$$

Equation (6) shows that the higher the rank of the mesh harmonic $k$, the lower is the maximum allowed speed fluctuation.

After band-pass filtering, the mesh harmonic $k$ is shifted to zero frequency, and the phase modulation $\phi_{k}(t)$ is extracted from the analytic signal and unwrapped. The instantaneous angular position of the shaft is then obtained by:

$$
\phi_{s h}(t)=\frac{\phi_{k}(t)}{k N_{s h}}+2 \pi \frac{f_{m}}{N_{s h}} t
$$

where $N_{s h}=N_{1}$ or $N_{2}$ depending on the shaft considered.

Notes:

- In model (1) $f_{m}$ is constant and so we are dealing with limited speed fluctuation only, especially this model is not appropriate for a ramp speed.

- This model is for one stage of the gearbox only. For a multistage gearbox, we then have the choice of the stage to use. This will be discussed later on an example (section 4.3).

- The transmission path effects (response of the structure) are not taken into account in the model. This can create additional phase modulations due to variation of the transmission path phase when the speed is fluctuating, as mentioned in [5]. However, if a structural resonance is located near the demodulated mesh component, its effect can be neglected if we assume the resonance frequency peak is wide compared to the demodulation bandwidth $2 B$. This is equivalent to assuming a damping factor of the resonance large compared to the relative speed fluctuation [6]. 


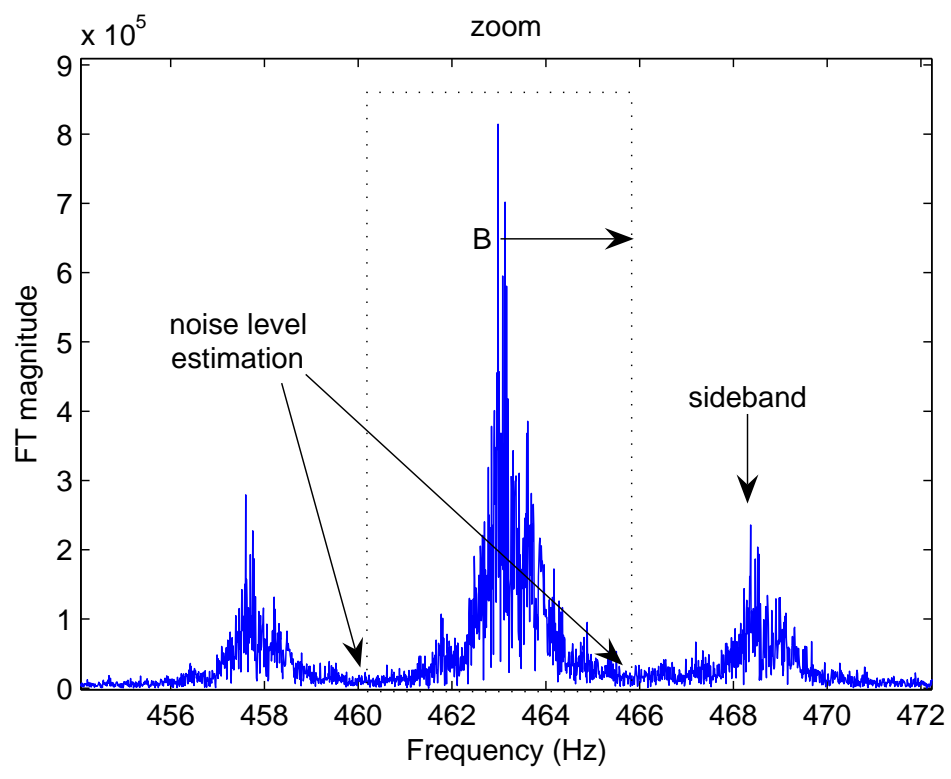

Fig. 1. Narrowband filtering of a mesh component of a gearbox for instantaneous speed estimation by phase demodulation.

\subsection{Phase estimation error}

For the speed estimation it was advised in [3] to try many different harmonics and to select the best one(s) based on a visual comparison between the estimated speeds. However this would not be a viable solution in a real time environment and we are looking here for an automated choice of the rank of the harmonic to use for demodulation. This choice must be performed in order to achieve the best angular precision of the recovered instantaneous position $\phi_{s h}(t)$. Eq. (7) shows that this precision is conditioned by the error on the phase modulation $\phi_{k}(t)$ estimated from mesh harmonic $k$. By writing the estimated phase $\hat{\phi}_{k}(t)$ as:

$$
\hat{\phi}_{k}(t)=\phi_{k}(t)+\delta \phi_{k}(t)
$$

where $\delta \phi_{k}(t)$ is the phase error on the estimated phase modulation, the standard deviation of the phase error can be expressed as (see appendix A):

$$
\sigma\left[\delta \phi_{k}\right]=\frac{1}{\sqrt{2}} 10^{-S N R_{k} / 20}
$$

where $S N R_{k}$ represents the local Signal to Noise Ratio (in dB) of mesh harmonic $k$ in the demodulated band of width $2 B$. This phase error $\delta \phi_{k}(t)$ then 
yields an error on the angular position of the shaft:

$$
\sigma\left[\delta \phi_{s h}\right]=\frac{\sigma\left[\delta \phi_{k}\right]}{k N_{s h}}
$$

Eq. (10) shows that the shaft phase error will be lowered for a gear with a higher number of teeth and also by using a higher rank for the demodulated mesh harmonic. However a high rank $k$ is also more restrictive for the maximum speed fluctuation allowed by eq. (6). Therefore care must be taken in the choice of the mesh harmonic.

In order to quantify the phase error from eq. (9), the local SNR of the demodulated mesh harmonic must be estimated. For the sake of simplicity we set the band-pass filter width to: $B=B_{\max }$ (eq. 5 ). The level of the noise power spectral density (PSD) is first estimated at both edges of the band-pass filter, as shown on figure 1 . For this estimation we suggest to use a bandwidth equal to $10 \%$ of the filter half bandwidth $B$. The noise is then assumed locally white inside the filter bandwidth with a level equal to the averaged PSD levels estimated at each edge of the filter. This provides an estimation of the noise power $P_{b}$ inside the filter bandwidth. Note that for this estimation we assume that no "parasite" component is included in the demodulation band (this case will be discussed on section 6 ). The total power $P_{\text {tot }}$ (signal + noise) in bandwidth $2 B$ is then estimated by integrating the PSD of the signal over this bandwidth. We thus obtain an estimation of the local SNR for each mesh harmonic $k$ by:

$$
S \hat{N} R_{k}=10 \log \left(\frac{P_{t o t}-P_{b}}{P_{b}}\right)
$$

This estimation provides an estimate of the shaft phase error (eq. $9 \& 10$ ).

\section{Estimation of the time synchronous averaged signal}

\subsection{Definition of the TSA of a shaft}

In [3] Bonnardot used the speed estimation in order to perform angular resampling of the signal. Our work is more specifically focused on the estimation of the time synchronous average (TSA), from which a number of diagnostic methods can be tackled out. In fact the TSA of a signal is a practical estimation of the signal average (first order cyclostationarity), which is defined in 
angular domain as:

$$
m_{x}(\theta)=E[x(\theta)]=m_{x}(\theta+\Theta)
$$

where $\Theta$ is the period of the cycle to which the machinery operation is periodic and $E[$.$] refers to the expectancy (ensemble average). Under the assump-$ tion of cycloergodicity, the ensemble average can be conveniently replaced by the cycle average (TSA) [7]. Note that gearboxes introduce different cycles related to each individual shaft. In this context signals have been named polycyclostationary because they are a combination of cyclostationary processes with different basic cycles [7].

\subsection{Angular resampling techniques}

In order to estimate the TSA, the time signal must be resampled according to the angle of rotation: this is known as the angular resampling or order tracking technique [8]. Different interpolation techniques have been proposed in the literature for resampling based on a tachometer or a shaft encoder $[8,9]$. Here this interpolation must be performed by using the shaft angular position $\phi_{s h}(t)$ estimated from one harmonic of the mesh vibration. In this purpose we consider the two following interpolation methods:

- Spline Angular Resampling (SAR): this is the method used in [3]. By using a 3rd order spline interpolation (cubic spline), this ensures a smooth interpolation function as its first two derivatives are continuous.

- Tacho Pulse Recovery (TPR): this is the alternative method proposed here. The tacho pulse instants $t_{p}(n)$, occurring once per revolution, are first recovered from the estimated shaft position inverse function by:

$$
t_{p}(n)=\phi_{s h}^{-1}(2 \pi n), \quad n=1, \ldots, N_{t o t}
$$

where $N_{t o t}$ is the integer number of shaft revolutions on the total duration of the signal. Then, by using Fourier interpolation [10] each shaft period is resampled up to a constant number of points between the recovered tacho instants $t_{p}(n)$.

Estimation of the tacho instants in eq. (13) requires to interpolate $N_{\text {tot }}$ points on the inverse function of the shaft instantaneous position $\phi_{s h}(t)$. This interpolation is not computationally demanding and moreover can be linear in practice since $\phi_{s h}(t)$ is slowly varying in time. It is however not the case for the interpolation of the signal versus angle (angular resampling), which is also more computationally demanding. 
For modelling the phase variations, the SAR method is a smoother interpolation (3rd order method), whereas the TPR method is piecewise linear. This can introduce some distortions in the resampled signal, however limited as the shaft speed is considered slowly varying within the shaft rotation period.

\subsection{Comparison and limitations in the real time computation}

The angular resampling scheme involves two stages: interpolation of the time signal and resampling according to the angle of rotation. Considering the real time objective of our methodology, the cost in time computation and in memory space is quite different for the two techniques presented in section 3.2 (TPR and SAR).

The TPR method being a piecewise method, each shaft period is resampled separately by using Fourier interpolation (Fourier transform, zero-padding in frequency domain and inverse Fourier transform [10]). Conversely, classical B-spline interpolation requires to solve a tridiagonal linear system and then to interpolate at least as many samples as in the original time signal (to avoid aliasing), which can be a highly computationally demanding task. Note that a running spline interpolator could be used for the online interpolation of the signal, which enjoys a significant computational advantage [11]. However this also needs to address the problem of online estimation of the shaft angular position, i.e. when each new data sample has been recorded.

In this paper the application is limited to the processing of a block of $\mathrm{N}$ samples of data only after these $\mathrm{N}$ samples have been recorded. Therefore, the algorithm is not completely real time as this needs to wait that the whole block duration (say 100s) has been recorded before being processed. However such a delay is acceptable for the application considered here. Nevertheless, the problem of real time angular resampling without speed sensor for online applications still needs to be considered (online tracking and filtering of a mesh harmonic, online estimation of the angular position).

The two angular resampling techniques will be compared in section 4.4 on a real case.

\subsection{Cut-off order of the estimated TSA}

The TSA estimation results from the instantaneous phase position of the shaft estimated in section 2 and, consequently, will be affected by the shaft phase error, which has been quantified from the local SNR in eq. (10). We aim here to quantify the effect of the phase error on the TSA estimation. 
It is known that errors on the estimated speed fluctuation will induce smearing of the higher shaft orders and, due to the averaging process, these higher orders will be lowered in the resulting TSA signal. The resulting low-pass filtering effect has been quantified in previous works and shown to correspond to the Fourier transform of the probability density function of the phase error $[7,12]$. A simple demonstration of this effect is given here in appendix B when considering only the phase errors of the recovered tacho pulses.

Let us assume that the density probability function of the shaft phase error $\delta \phi_{s h}(t)$ can be modelled as a Gaussian function (see section 4 for justification), with zero mean and standard deviation $\sigma\left[\delta \phi_{s h}\right]$ given by eq. (10). This error being expressed as a phase (in radians), it must be related to the jitter error of the recovered tacho pulses on each shaft period, which is: $\sigma\left[\delta \phi_{s h}\right] /(2 \pi)$ shaft period. The corresponding low-pass filter (in order domain) will also have a Gaussian shape with a $3 \mathrm{~dB}$ cut-off order equal to: $\sqrt{\ln 2} / \sigma\left[\delta \phi_{s h}\right] \simeq$ $0.83 / \sigma\left[\delta \phi_{s h}\right]$. Thus we define the $3 \mathrm{~dB}$ cut-off order $o_{c}^{(k)}$ of the TSA signal estimated from mesh harmonic $k$ as:

$$
o_{c}^{(k)}=\frac{0.83}{\sigma\left[\delta \phi_{s h}\right]}=0.83 \sqrt{2} k N_{s h} 10^{S N R_{k} / 20} \quad \text { (orders) }
$$

Cut-off order $o_{c}^{(k)}$ represents the maximum "resolution" in terms of shaft orders recovered in the estimated TSA signal, and the ratio $o_{c}^{(k)} / N_{s h}$ represents the resolution in terms of the number of recovered mesh harmonics. Eq. (14) shows that this ratio depends on two parameters: the rank $k$ and the $S N R_{k}$ of the mesh harmonic $k$ selected for speed estimation. Shaft orders above the cut-off order are expected to be affected by phase errors and so will be lowered by the averaging process. This effect will be illustrated in next section on a real case.

\section{Real case study}

The gearbox under study is a two-stage helical reduction gearbox with ratio 20.5:1 designed by WYKO Fenner UK and here used in a wastewater treatment installation. It is driven by a $1.5 \mathrm{~kW}$ motor. The output shaft speed is about 70rpm. Fig. 2 shows the gearbox under experiment as well as a simple schematic of the gear components with their respective number of teeth. An optical pick-up sensor was used as a tachometer for measurement of the output shaft speed. This speed measurement was used in a first instance in order to validate the methodology based on TSA. Experimental conditions are however not ideal in this outdoor environment for installation of the speed sensor. The metallic protection covering the chain driving the paddle system had to be removed, and consequently the reflective tape stuck onto the output 
shaft could be easily moistened and the measurement affected. Thus it would be advantageous for future trials to avoid the need of this speed measurement.
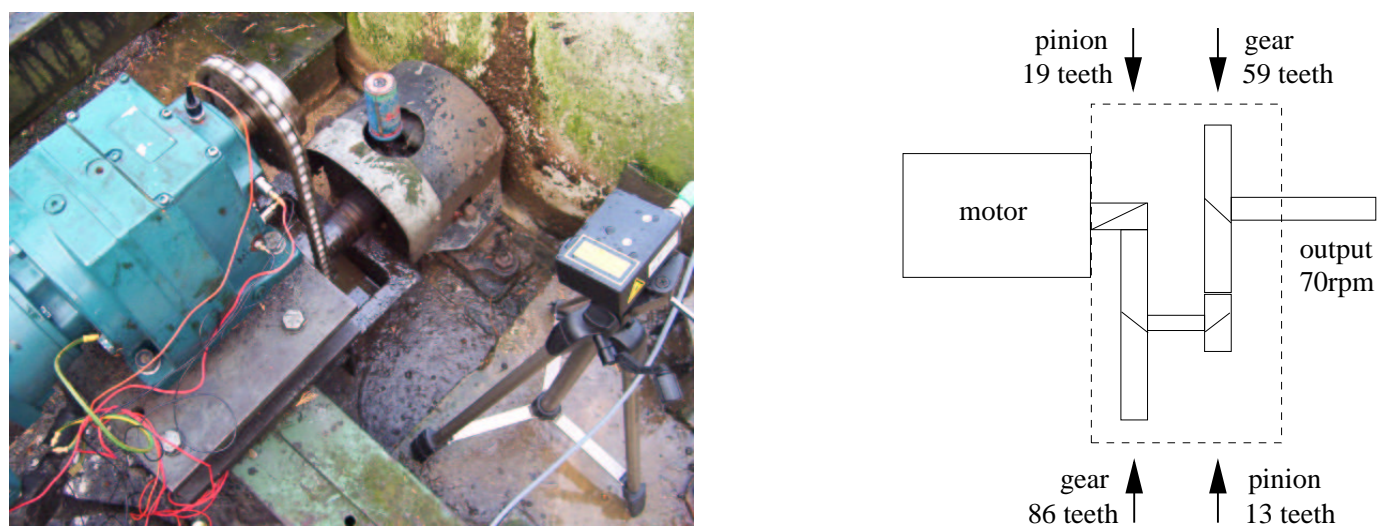

Fig. 2. Two-stage helical reduction gearbox used in a wastewater treatment site (left) and simple schematic (right).

Fig. 3 shows the Power Spectral Density (PSD) in the low frequency part of the vertical acceleration signal measured on the casing near the output bearing (see figure 2). The first three harmonics of the output stage mesh (at $\simeq 69 \mathrm{~Hz}$ ) are indicated. Some electrical components can be observed at 50 and $100 \mathrm{~Hz}$. The strong component at about $30 \mathrm{~Hz}$ is related to the load (chain driven by the gearbox). Although the highest mesh harmonic is here the second one, it appears to be also the noisiest of the three (Tab. 1 lists the local SNR estimated for each component).

\subsection{Phase error of the recovered tacho pulses}

The tachometer signal will be used here as a reference for the comparison between the recovered tacho pulse instants with the measured ones. The harmonics of the output stage mesh are first band-pass filtered with a bandwidth $2 B$ equal to the lowest shaft speed (here the output shaft at $1.2 \mathrm{~Hz}$ ). This bandwidth is chosen in order to allow a maximum speed fluctuation, i.e. $B=B_{\max }=0.6 \mathrm{~Hz}$ (eq. 5 ).

The estimated and maximum allowed speed fluctuations are shown in table 1 for the first three mesh harmonics (for higher harmonics the estimated speed fluctuation becomes higher than the allowed one and so may be affected by unwrapping errors). Phase demodulation of each one of the mesh harmonics yields an estimation of the shaft angular position. Fig. 4 shows the analytic signal obtained after band-pass filtering and demodulation of the first two mesh harmonics, performed by selection of the part of the Fourier transform and then translation to zero frequency. The second harmonic appears much noisier than the first one here. The tacho instants are then recovered and 


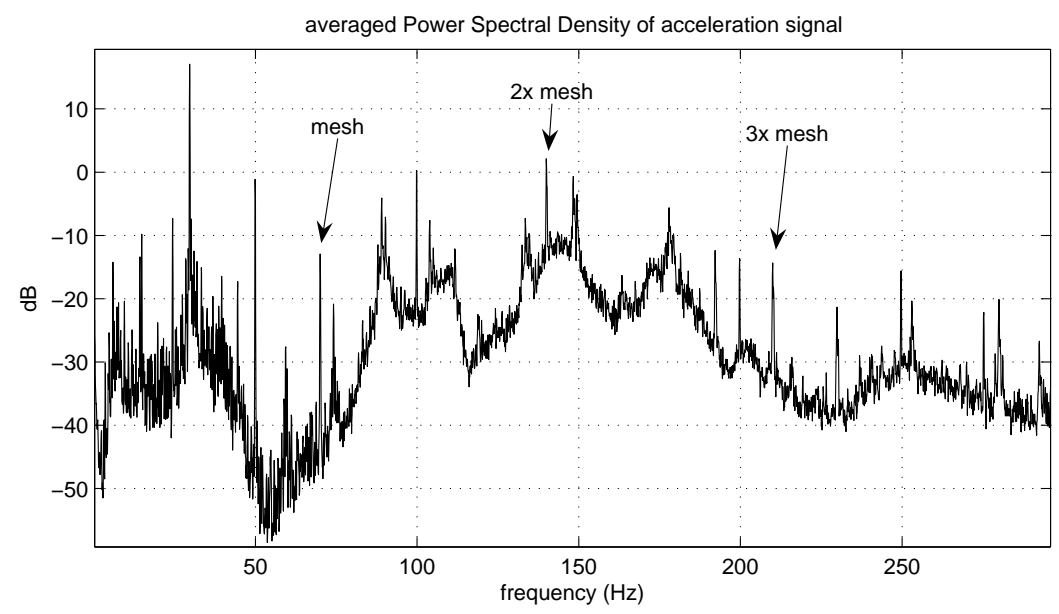

Fig. 3. Low frequency part of the spectrum of the vertical acceleration measured on the gearbox near the output bearing. The first three harmonics of the output stage mesh $(\simeq 69 \mathrm{~Hz})$ are indicated.

the tacho phase errors are computed as the difference between the measured and recovered tacho instants (with time origin taken at the first measured tacho pulse). The distributions of the tacho errors computed on a 200s signal duration (about 230 rotations of the output shaft) are shown on fig. 5 for the first two mesh harmonics. Note that the Gaussian assumption regarding the shape of the error distribution made in section 3.4 seems to be reasonable here.
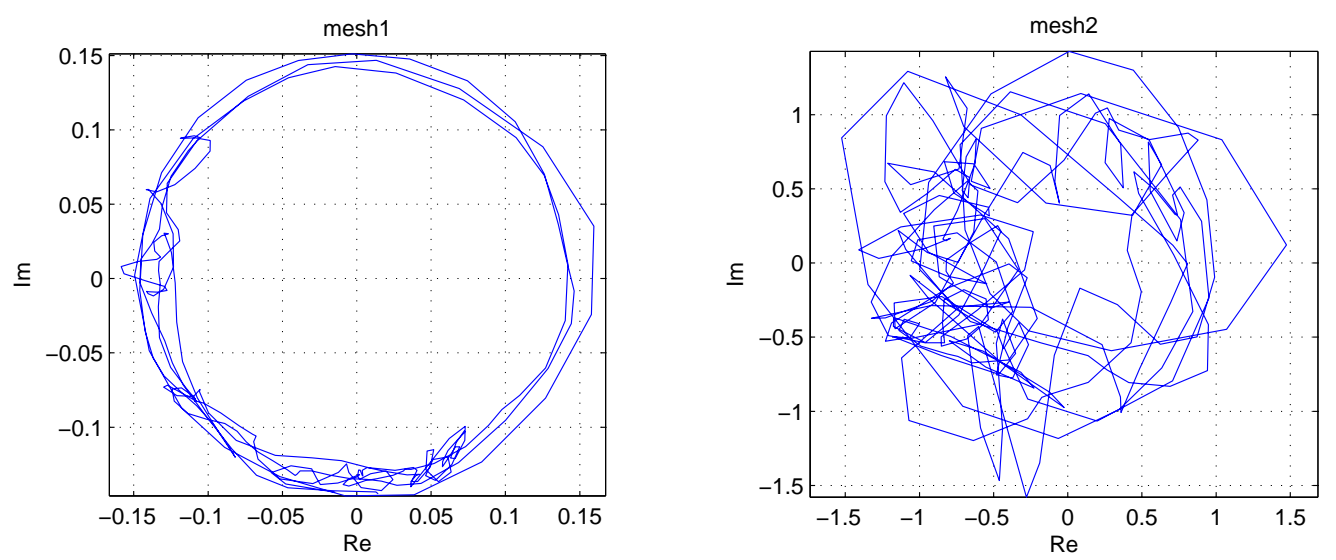

Fig. 4. Representation of the demodulated analytic signal in the complex plane from: first mesh harmonic (left), second mesh harmonic (right).

The experimental tacho phase error can now be compared with the phase error standard deviation predicted from the local SNR of the mesh harmonics (eq. 9). Results in Tab. 1 show that the standard deviation of the predicted phase error is relatively well in accordance with the experimental one for the first three harmonics of the output stage mesh. This result validates the phase error estimation based on the local SNR of the mesh harmonic. 

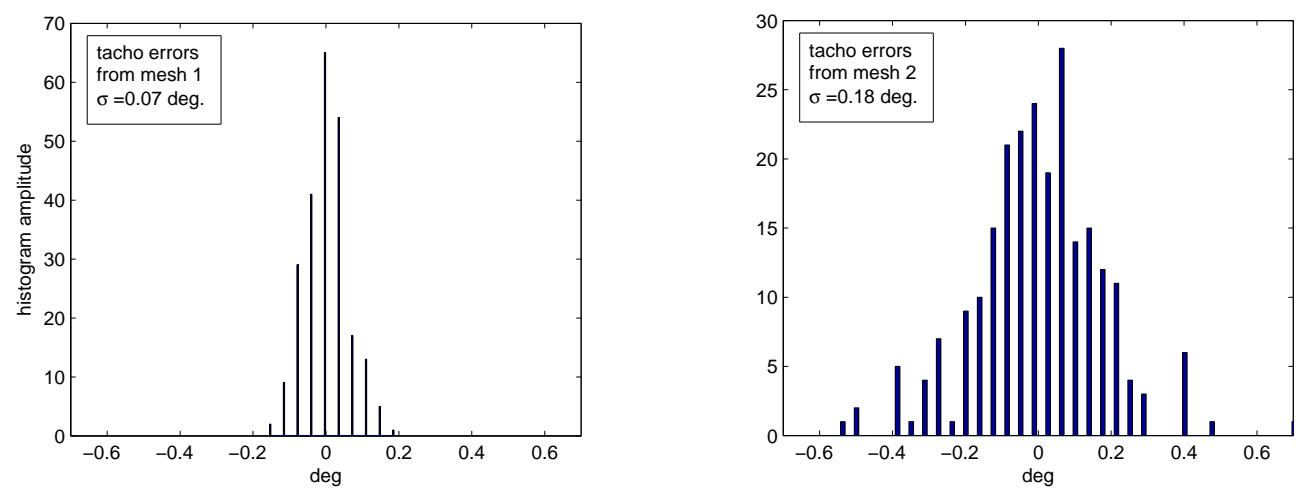

Fig. 5. Distributions of the tacho phase errors computed as the difference between the measured (from tachometer) and recovered tacho pulse instants from phase demodulation of the output stage mesh: first harmonic (left) and second harmonic (right).

Table 1

Local SNR and speed fluctuation estimated for each mesh components and comparison of the predicted and measured phase errors for the output shaft.

\begin{tabular}{|c|c|c|c|c|}
\hline \multirow[b]{2}{*}{ Mesh harmonic demodulated } & \multicolumn{3}{|c|}{ output stage } & \multirow{2}{*}{$\begin{array}{c}\text { input stage } \\
1 \text { st }\end{array}$} \\
\hline & 1 st & 2nd & 3rd & \\
\hline estimated local SNR (dB) & 22 & 7 & 13 & 17 \\
\hline cut-off order of the TSA & 970 & 320 & 920 & 3220 \\
\hline estimated speed fluctuation (\%) & 0.19 & 0.38 & 0.23 & 0.31 \\
\hline maximum allowed fluctuation (\%) & 0.85 & 0.42 & 0.28 & 0.58 \\
\hline \multicolumn{5}{|l|}{ Output shaft phase error (std dev. in degree) } \\
\hline predicted from local SNR & 0.048 & 0.145 & 0.051 & 0.015 \\
\hline measured from tacho signal & 0.068 & 0.166 & 0.096 & 0.057 \\
\hline
\end{tabular}

\subsection{Comparison of the estimated TSA}

The TSA signal is now computed for the three mesh harmonics of the output stage after resampling by using the TPR interpolation method (section 3.2). The TSA is affected by the phase error by a low-pass filter which $3 \mathrm{~dB}$ cutoff order, defined by eq. (14) in section 3.4, is inversely proportional to the phase error standard deviation. Fig. 6 compares the spectra of the TSA signals (obtained by Fourier transform of the TSA) estimated from tacho signal and the first two mesh harmonics. Although the two TSA spectra for tacho and mesh fundamental appear very similar, the low-pass filtering effect is clearly observed when using 2nd mesh harmonic (due to a lower local SNR of this component): on this spectrum the higher mesh harmonics (rounds) are low- 

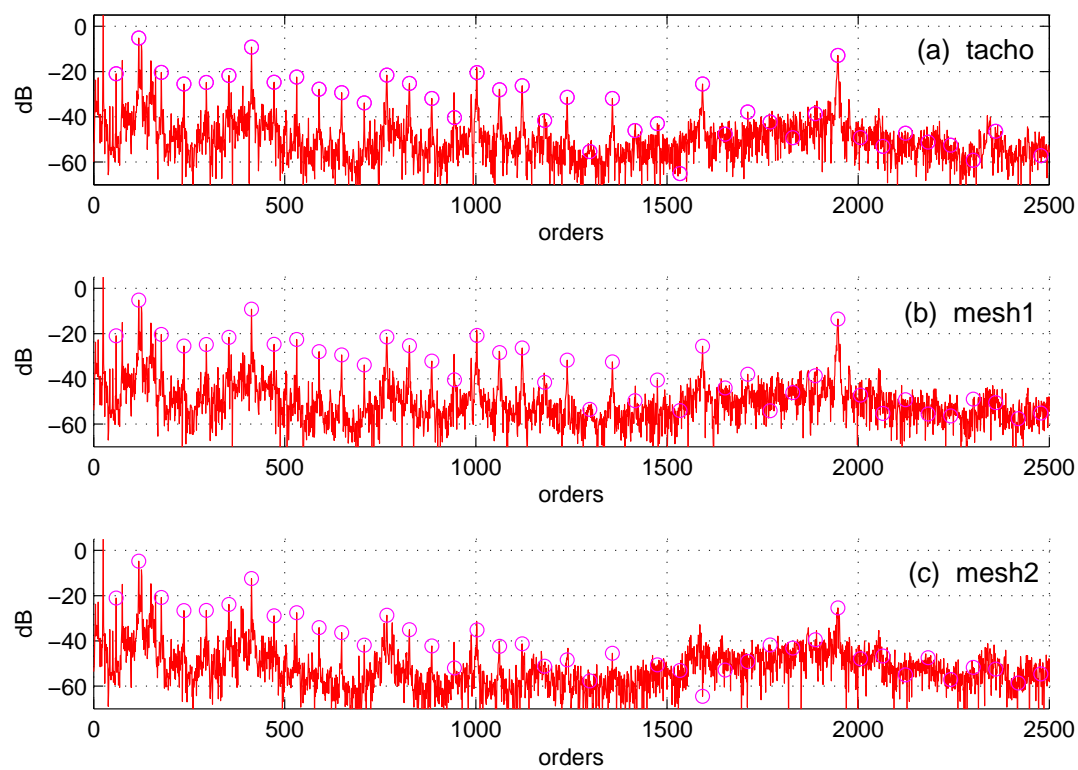

Fig. 6. Comparison of order spectra of the output shaft TSA estimates: (a) from tacho signal, (b) from mesh fundamental (cut-off order $o_{c}^{(1)}=970$ ) and (c) from mesh 2 nd harmonic of output stage $\left(o_{c}^{(2)}=320\right)$. The rounds indicate the output stage mesh harmonics (59 multiple orders).

ered compared to the other two spectra. The cut-off order estimated for 2nd harmonic (320) seems in accordance with the low-pass filtering effect observed on the spectrum.

\subsection{Use of the input stage mesh}

The speed estimation can also be performed by using the input stage mesh between the motor and inner shafts of the gearbox, running at about $450 \mathrm{~Hz}$ (see fig. 2). In this case however only the mesh fundamental can be used since from second harmonic the estimated speed fluctuation becomes higher than the maximum allowed one by eq. (6).

\subsection{1 for estimating the output shaft TSA}

In this case, in order to recover the output shaft angular position $\phi_{\text {out }}(t)$, the phase modulation of the input mesh fundamental is first divided by the number of teeth of the inner shaft gear (86), which provides the instantaneous angular position of this shaft: $\phi_{i n}(t)$, and then further divided by the gear ratio $R$ of the output stage $(R=59 / 13 \simeq 4.54)$. As a consequence the phase error of the output shaft should in theory be $R$ times lower than that of the inner shaft. 
The phase error standard deviation estimated from the local SNR of the input mesh frequency is $0.068 \mathrm{deg}$. for the inner shaft, which gives an theoretical error of $0.015 \mathrm{deg}$. for the output shaft. However, the experimental error obtained by comparison of the recovered tacho pulses with those of the measured tacho signal is almost 4 times higher (see table 1). This brings to the conclusion that the phase error of one shaft cannot simply be divided by the gear ratio of the stage to estimate the phase error on the other shaft. This is likely to be due to the transmission errors in the meshing process (static transmission error, tooth deflection, torsional vibrations of the shafts) which are not taken into account here in the model. A better estimation of the output phase error would require further modelling of these effects, which is out of the scope of this paper. However the experimental phase error obtained from the input stage is still lower than that of the output stage, whatever harmonics. This comes from the fact that we use a higher frequency component, so that the phase errors in the transmission are compensated by a better accuracy of the demodulated phase.

\subsection{2 for estimating the inner shaft TSA}

For estimation of the inner shaft angular position and TSA, the input stage is more advantageous than the output stage (in the case of a reduction gearbox). By using the output stage the phase error for this shaft is $0.24 \mathrm{deg}$. (with 1 st harmonic), corresponding to a cut-off order of about 200, whereas by using the input stage the error is reduced to 0.068deg. (690 orders). The effect on the TSA signal can be observed on figure 7: the spectrum contains higher frequency components when using the input stage compared with the output stage. The low-pass filtering effect can also be observed on the TSA signal. This difference in phase error is mainly due to rather different number of teeth of the inner shaft gears at the input and output stages (86 and 13 respectively). Thus when two wheels are connected to a same shaft, the one with the higher number of teeth, i.e. with the higher mesh frequency, should be preferred (similarly to a shaft encoder).

In conclusion, the use of the input stage mesh performs well for both the output and inner shafts. It is also obviously preferable to use for the TSA of the motor shaft. However the maximum allowed speed fluctuation is lower here because the input stage gear has a higher number of teeth than the one at the output stage (see table 1 ). 


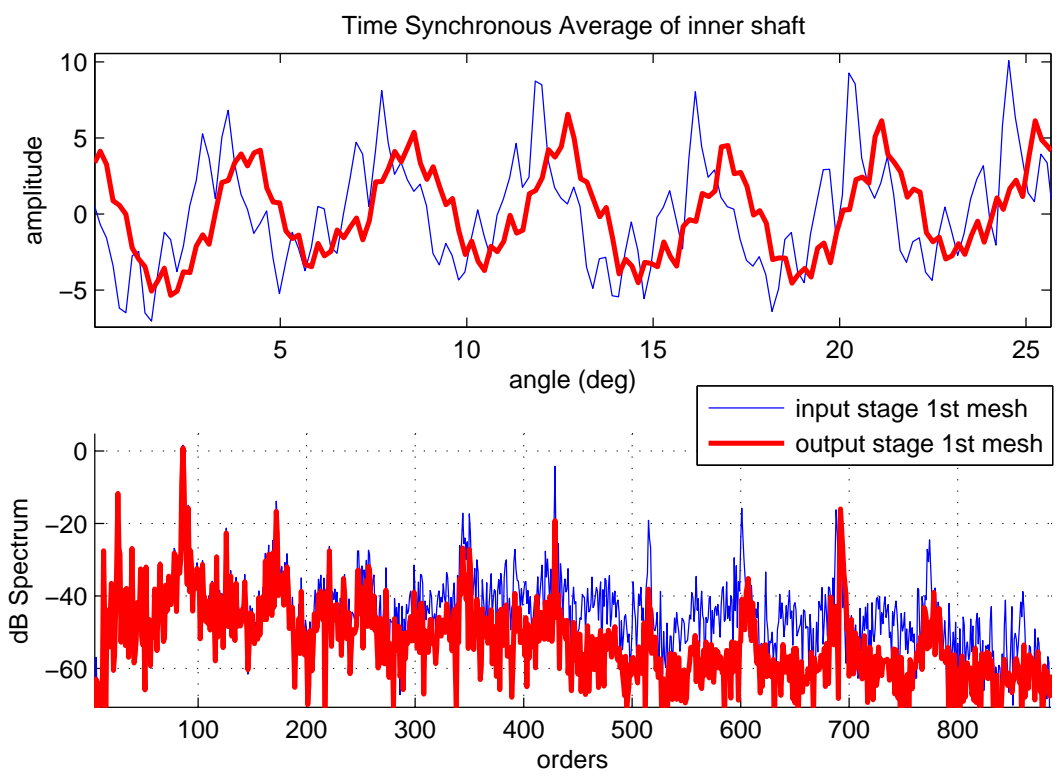

Fig. 7. Estimated TSA signals for the inner shaft of the gearbox (500 averages) with speed estimated from the input stage mesh (thin curve) and output stage mesh (thick curve).

\subsection{Comparison of the Tacho Pulse Recovery and Spline interpolation meth- ods}

The TSA signals in previous sections were estimated by using the Tacho Pulse Recovery (TPR) method presented in section 3.2. In this section we compare results when using the Spline Angular Resampling (SAR) method [3]. Fig. 8 shows the interpolated tacho pulses of the TPR method on the estimated shaft phase fluctuation. Although this method assumes a piecewise linear model of the phase fluctuation, the difference between the model and the real phase fluctuation remains small because the estimated phase is slowly varying, so that we can expect the TSA signal not to be too affected by this approximation.

\subsection{1 in the rate of convergence of the TSA}

In order to compare the TSA signals for both interpolation methods, we observe how the TSA energy decreases versus the number of averages $n$. For a purely random signal, the energy decreases as $1 / n$, thus forming a straight line in a log-log diagram. For a gearbox signal the situation is more complex since the decrease is not as regular due to interferences from other shaft components [13]. However when all random and non-synchronous components have been averaged out the TSA energy should stop decreasing and converge to a constant value, ideally represented by a horizontal line in the diagram (note that this method could be an empirical way to estimate the required minimum 


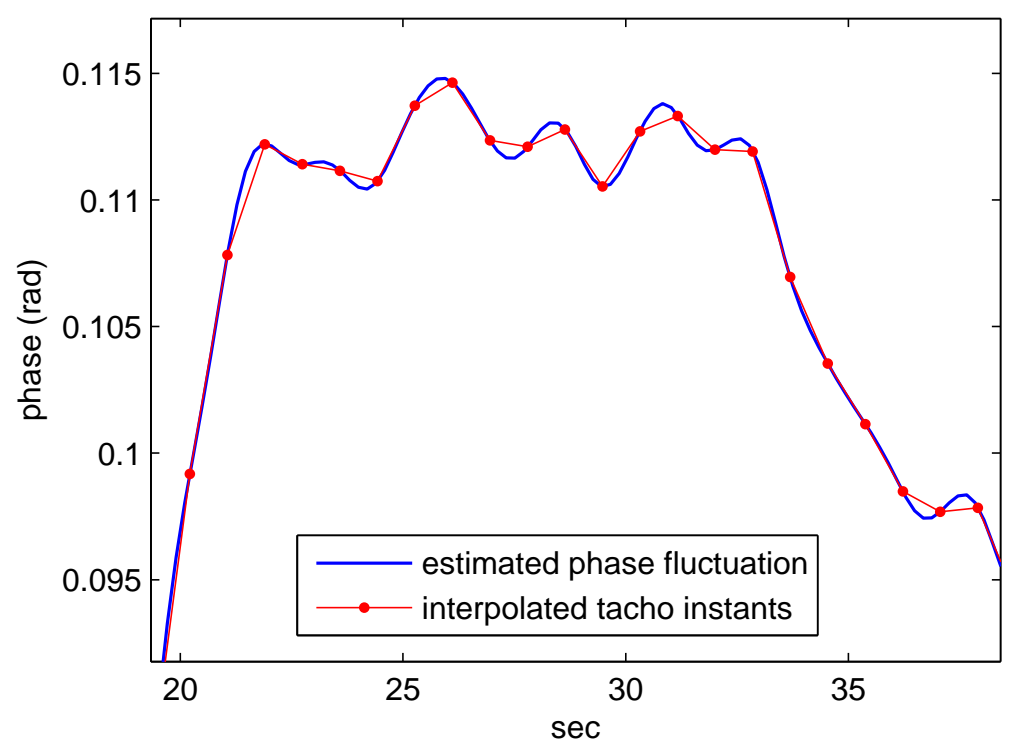

Fig. 8. Estimated shaft phase fluctuation and recovered tacho pulse instants.

number of averages, but this is not further developed here).

Fig. 9 shows the decrease of the output shaft TSA energy versus $n$ for both interpolation methods when using the output stage mesh fundamental. On the left figure the convergence is assessed to be reached at about 100 averages and the final value is almost identical for both methods (0.03dB difference). On the right figure the signal has first been low-pass filtered with a cut-off frequency corresponding to the estimated cut-off order for this mesh component (970 orders). In this case no significant difference appears between the two methods. Note the convergence seems faster with prior low-pass filtering: this is due to the fact that orders higher than the cut-off order are affected by the phase error (the remaining jitter effect) so that their amplitudes in the TSA are slowly decreasing with the averaging process.

\subsection{2 in the computation time}

We now compare in table 2 the computation time for both methods. As expected in section 3.3 the SAR method appears to be more demanding in time and in memory space than the TPR method. Indeed for a long signal duration (300s) the usual spline resampling function running under Matlab on a standard PC could not be computed due to a memory error, whereas for the TPR method the computation time remains small and increases in a more linear fashion. Therefore we argue that the TPR method should be preferred in a real time environment due to its gain in simplicity and in computation cost and its efficiency. 

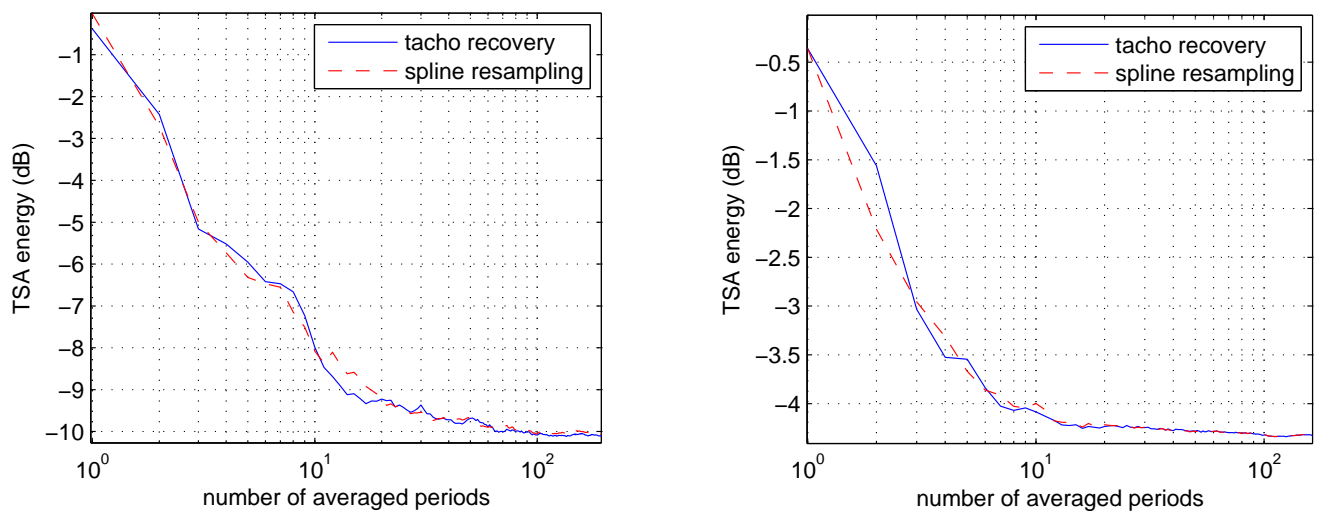

Fig. 9. Decrease of the output shaft TSA energy versus the number of averages for the tacho recovery and spline resampling interpolation methods: full range frequency spectrum (left), range limited to cut-off order 970 (right). No significant difference appears in the TSA energy rate of convergence between the two methods.

Table 2

Computation time of the output shaft TSA for both interpolation methods and for different signal lengths (100s representing 1125000 samples or 118 shaft rotations). Programs are running under Matlab on a PC with $3 \mathrm{GHz}$ clock speed and $1 \mathrm{Go}$ memory.

\begin{tabular}{|l|llll|}
\hline Interpolation method & signal duration: & $100 \mathrm{~s}$ & $200 \mathrm{~s}$ & $300 \mathrm{~s}$ \\
\hline Tacho Pulse Recovery & computation time: & $3 \mathrm{~s}$ & $4 \mathrm{~s}$ & $5 \mathrm{~s}$ \\
Spline Angular Resampling & & $8 \mathrm{~s}$ & $40 \mathrm{~s}$ & pb memory \\
\hline
\end{tabular}

\section{Proposed methodology for estimation of the time synchronous average without speed sensor}

Based on the previous theoretical developments and experimental study we now propose a complete methodology for the estimation of the TSA signal of a particular shaft in a gearbox by speed estimation from the mesh vibration. Figure 10 presents the flow chart of the proposed methodology. Each step is now detailed in the following.

The method first requires an a priori value of the shaft speed and the number of teeth of the two gears in mesh at the considered stage. The mesh frequency is then adjusted to its actual value by a peak detection performed on the signal spectrum in a certain frequency range around the a priori mesh frequency (eg. $\pm 5 \%$ ). In order to avoid spurious maxima, the spectrum is first smoothed by convolution with a narrow window (we used a Parzen window with a 9 frequency bin width). Also, for a better robustness in the detection, we recommend to use several mesh harmonics by accumulating the spectrum amplitudes at multiples of the search frequency (eg. 3 harmonics). 
The next step is to estimate the local SNRs for a set of mesh harmonics (eq. 11). The filter half bandwidth is set to half the slowest gear speed in order to allow maximum speed fluctuation (eq. 5). The cut-off order for each harmonic is then computed via eq. (14). The selected harmonic at this stage will be that with the highest cut-off order for the TSA signal (or with the lowest phase error of the estimated speed). We performed this selection among the five first harmonics only since higher harmonics generally have a lower SNR.

The previously selected harmonic is then demodulated and the shaft angular position is estimated (eq. 7). Before angular resampling, a test is performed on the estimated speed: the peak-to-peak speed fluctuation must be lower than the maximum allowed one (eq. 6). If this is not the case it means the estimated speed is not accurately estimated (unwrapping problems may have occurred) and a lower rank harmonic is selected and then again demodulated. This scheme is performed as long as the speed test is not satisfied. If even the first harmonic does not pass the test it means the speed fluctuation are too

high for the method to perform. In that case the use of another stage can be considered.

Once the shaft instantaneous position has been estimated, the tacho pulse instants are recovered and each rotation period is resampled up to a constant number of samples (which must be higher than the highest number of samples per period in order to avoid aliasing). The average of all resampled shaft periods provides the TSA signal for the considered shaft.

It should be noted that the proposed methodology applies for one stage of the gearbox only. The choice of the stage will depend on the application considered: if we are interested in monitoring the output gear only then the output stage should be preferred since we have here a higher allowed speed fluctuation and also a good agreement between the experimental and predicted phase errors (Tab. 1). However if all gears need to be monitored in the gearbox then all TSA signals of all shafts need to be estimated. In that case the input stage should be preferred as shown in section 4.3. It is even possible to perform only one angular resampling and then to estimate the TSA of each shaft by extracting the appropriate components in frequency domain [7].

\section{Discussion}

A particular problem mentioned in [3] can arise when a "parasite" component is included in the demodulation bandwidth of a particular mesh harmonic. The effect of the parasite component will be first to decrease the local SNR and so to lower the cut-off order for this harmonic, and second to create an additional strong fluctuation in the estimated speed, so that it is likely that 


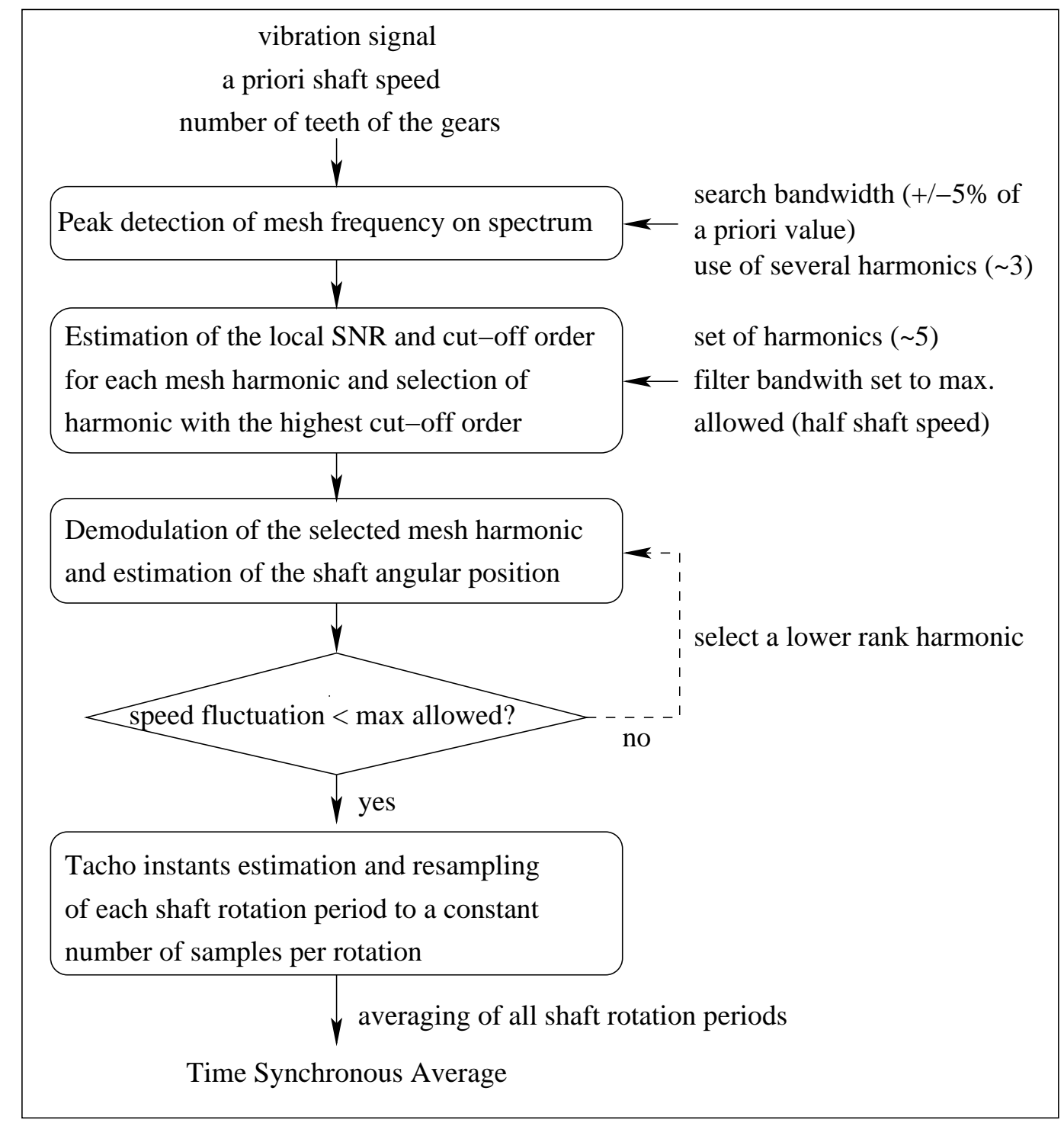

Fig. 10. Methodology for estimation of the TSA of a particular shaft in a gearbox without speed sensor.

the mesh harmonic will not pass the speed fluctuation test in that case.

Table 3

Selection of input stage mesh harmonic for estimating the inner shaft TSA. The third harmonic is here selected (highest cut-off order). The SNR of first harmonic is low here due to a parasite component close to this mesh.

\begin{tabular}{|l|lllll|}
\hline mesh harmonic & 1 & 2 & 3 & 4 & 5 \\
\hline local SNR $(\mathrm{dB})$ & -1.4 & 13.5 & 13.9 & 1.1 & 8.9 \\
cut-off order & 84 & 938 & 1478 & 448 & 1376 \\
\hline
\end{tabular}

Table 3 presents an interesting case when estimating the inner shaft TSA for a different type of load coupled to the same gearbox. In this case the input stage is used and the mesh frequency is estimated at $452.5 \mathrm{~Hz}$ after peak detection. 

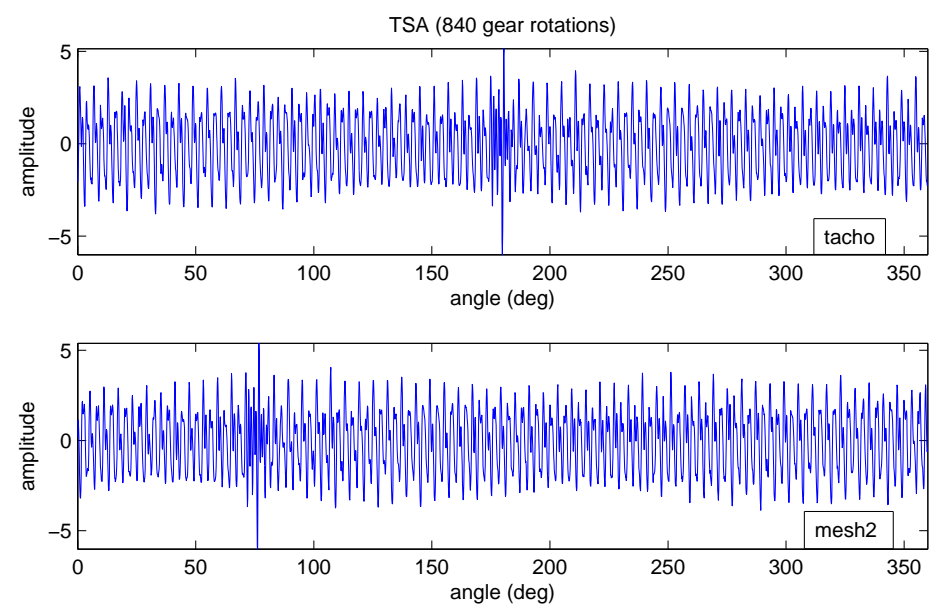

Fig. 11. Estimated TSA from tachometer (top) and from mesh second harmonic (bottom) for a spur gearbox with a simulated 10\% surface pitting on one tooth of the gear. A time shift appears between the two TSA as the zero angle position is undetermined when using the proposed method for speed recovery.

The filter half bandwidth is set to $B_{\max }=2.7 \mathrm{~Hz}$ (inner shaft speed is $5.4 \mathrm{~Hz}$ ). The local SNRs and the cut-off orders are estimated for the first five mesh harmonics (see table 3). The third harmonic is here selected due to its highest cut-off order. Note that the first harmonic SNR is abnormally low here: an inspection of the spectrum reveals an electrical component at $450 \mathrm{~Hz}$ which is inside the demodulation bandwidth of this harmonic. This parasite component is the reason for this low SNR. The estimated speed fluctuation for the third harmonic is $0.11 \%$ ( $0.19 \%$ max.). By using first harmonic it would have been $0.33 \%$ due to the parasite, which is however still in the allowed range $(0.58 \%)$. Thus in this particular case the parasite is discarded by the SNR estimation rather than by the test on the speed fluctuation.

The proposed methodology has also been tested with success on other types of industrial gearboxes, especially with spur gears. However the helical gearbox presented here was a more difficult case since the mesh harmonics were found to be lower than that of the spur gears. The tested gearboxes being used under relatively steady loading conditions, we have not found so far cases where the speed fluctuation limitation prevented the method to perform. It should be mentioned however that one drawback of the method is that, contrary to a tachometer, we have obviously no more angular reference available here for the localisation of a fault on the gear, as shown on figure 11. 


\section{Conclusion}

The purpose of this paper was to extend the original idea proposed in [3] for angular resampling by using the mesh vibration of a gearbox and to propose a general methodology that could be used as an automated scheme for condition monitoring of gear systems without the need of a speed sensor. First the angular precision of the shaft has been quantified by the phase error standard deviation, which is related to the local signal/noise ratio (SNR) of the demodulated mesh harmonic. By assuming a normal distribution of the phase error, the corresponding low-pass filtering effect on the TSA can be quantified by its $3 \mathrm{~dB}$ cut-off order, which depends on both the rank and the local SNR of the selected mesh harmonic. Comparison on a real case shows good agreement between the predicted and measured (from a tachometer) phase errors of the estimated shaft angular position, and the filtering effect on the TSA signal has also been observed for some mesh harmonics. Furthermore an alternative method for angular resampling of the signal from the estimated speed has been proposed: the Tacho Pulse instants Recovery. This method showed no significant difference compared to the spline interpolation method. Its advantage is to be much less demanding in computation time and in memory space, especially for relatively long signal duration, and is thus better adapted to real time applications. Finally a methodology has been proposed for estimation of the TSA without speed sensor, which requires only an a priori estimation of the shaft speed and the number of teeth of the meshing gears. The choice of the stage to use has been discussed and will generally depend on the application considered. The proposed methodology forms an automated scheme which could be easily implemented and can be useful for condition monitoring applications where speed measurement is not advisable due to difficult environmental conditions. One drawback of the method is obviously that no more angular reference is available for the TSA for the localisation of a fault on the gear.

\section{Acknowledgements}

This work is a part of a project funded by the DTI (UK). The authors gratefully acknowledge the financial support of the DTI, grant STI/6/037. We also gratefully thank industrial partners for their in-kind support: WYKO (Mr. Ohren, Mr. Patel), Severn Trent Water (Mr. Stanley), and Compact Orbital Gears (Mr. LaPayne, Mr. Huges). 


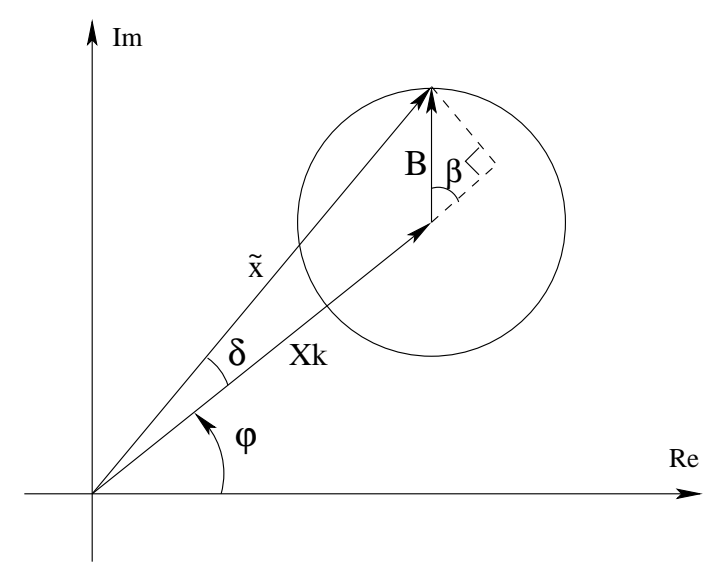

Fig. A.1. Representation of the analytic signal in the complex plane and of the phase error $\delta$.

\section{A Relation between phase error and local SNR in the demodula- tion band}

The signal from model (1) after band-pass filtering of mesh harmonic $k$ and frequency shift to zero is written

$$
x(t)=X_{k} \cos \varphi_{k}(t)+b(t)
$$

where $\varphi_{k}(t)=\phi_{k}(t)+\Phi_{k}$ is the phase modulation to be recovered and $b(t)$ is the noise present in the filter bandwidth, assumed zero mean and with variance $\sigma_{b}^{2}$. After applying the Hilbert transform, we obtain the analytic signal:

$$
\tilde{x}=X_{k} e^{j \varphi_{k}}+B e^{j \beta}
$$

with $j^{2}=-1$, the time dependency being omitted here. In eq. (A.2) $X_{k}$ is a constant, $\varphi_{k}$ is a deterministic function of time, $B$ and $\beta$ are random and represent the analytic function of noise $b(t)$ (so that $b=B \cos \beta$ ). By assuming $\beta$ uniformly distributed in interval $[-\pi, \pi]$ the variance of noise $b$ is

$$
\sigma_{b}^{2}=E\left[|B \cos \beta|^{2}\right]=\sigma_{B}^{2} / 2
$$

The signal to noise ratio (SNR) in $\mathrm{dB}$ of demodulated signal $x$ is written

$$
S N R=10 \log \frac{X_{k}^{2} / 2}{\sigma_{b}^{2}}=20 \log \frac{X_{k}}{\sigma_{B}}
$$

The analytic signal $\tilde{x}$ is represented as a vector in the complex plane on figure A.1. At one given instant $t$, the effect of the additive noise of amplitude $B$ will 
be to create a phase error $\delta$ on the estimated phase $\varphi$. The resulting position of the analytic signal is located on a circle of radius $B$, all points on this circle being equiprobable since angle $\beta$ is assumed randomly distributed. From this figure we can write the trigonometric relation:

$$
\tan \delta=\frac{B \sin \beta}{X_{k}+B \cos \beta}
$$

By assuming a relatively good SNR, the noise amplitude $B$ remains small compared to $X_{k}$, so that (A.5) can be simplified by

$$
\tan \delta \simeq \delta \simeq \frac{B \sin \beta}{X_{k}}
$$

The mean phase error of $\delta$ is zero. The variance is

$$
\sigma[\delta]^{2}=\frac{E\left[B^{2} \sin ^{2} \beta\right]}{X_{k}^{2}}=\frac{\sigma_{B}^{2}}{2 X_{k}^{2}}
$$

since $E\left[\sin ^{2} \beta\right]=1 / 2$. Thus the phase error standard deviation is related to the local SNR at mesh harmonic $k$ by

$$
\sigma[\delta]=\frac{1}{\sqrt{2}} 10^{-S N R / 20}
$$

\section{B Effect of the tacho phase error on the time synchronous average}

Due to the phase error of the shaft angular position estimation, the tacho pulse instant at rotation $i$ is estimated with an jitter error $\epsilon_{i}$. After angular resampling, the shaft $i$ th rotation period $x_{i}(t)$ can be written

$$
x_{i}(t)=s\left(t-\epsilon_{i}\right)+b_{i}(t)
$$

where $s(t)$ is the deterministic part of the signal which is periodic with the rotation period of interest and $b(t)$ represents the random and non-synchronous sources. The "ideal" time synchronous averaged (TSA) signal, i.e. after sufficient number of averaging, is

$$
m_{x}(t)=E\left[x_{i}(t)\right]=E\left[s\left(t-\epsilon_{i}\right)\right]
$$

The TSA being exactly periodic, its spectrum (orders) is simply computed by a Fourier transform. Assuming commutativity with expectancy (linear opera- 
tors) we get:

$$
M_{x}(f)=F T\left\{m_{x}(t)\right\}=E\left[S(f) e^{-j 2 \pi f \epsilon_{i}}\right]
$$

where $S(f)=F T\{s(t)\}$ is deterministic. By denoting $p\left(\epsilon_{i}\right)$ the probability density function of random variable $\epsilon_{i}$, we obtain:

$$
M_{x}(f)=S(f) \cdot E\left[e^{-j 2 \pi f \epsilon_{i}}\right]=S(f) \cdot \int e^{-j 2 \pi f \epsilon_{i}} p\left(\epsilon_{i}\right) d \epsilon_{i}=S(f) . \Phi(f)
$$

where $\Phi(f)$ represents the Fourier transform of $p\left(\epsilon_{i}\right)$, which acts as a low-pass filter on the TSA spectrum. The narrower is $p\left(\epsilon_{i}\right)$ (the lower is the phase error), the higher is the cut-off frequency (or cut-off order) of filter $\Phi(f)$ and the more orders of signal $s(t)$ can be recovered in the TSA.

\section{References}

[1] S. Braun, Acustica Vol. 32 (1975) 69-77. The extraction of periodic waveforms by time domain averaging.

[2] P.D. McFadden, Mechanical Systems and Signal Processing 1 (1) (1987) 83-95. A revised model for the extraction of periodic waveforms by time-domain averaging.

[3] F. Bonnardot, M. El Badaoui, R.B. Randall, J. Danière, F. Guillet, Mechanical Systems and Signal Processing 19 (2005) 766-785. Use of the acceleration signal of a gearbox in order to perform angular resampling (with limited speed fluctuation).

[4] P.D. McFadden, Journal of Vibration, Acoustics, Stress, and Reliability in Design 108 (1986), 165-170. Detecting fatigue cracks in gears by amplitude and phase demodulation of the meshing vibration.

[5] C.J. Stander, P.S. Heyns, Mechanical Systems and Signal Processing 20 (2006) 1511-1522. Transmission path phase compensation for gear monitoring under fluctuating load conditions.

[6] F. Combet, P. Jaussaud, N. Martin, Mechanical Systems and Signal Processing 19 (2005) 239-257. Estimation of slight speed gaps between signals via the scale transform.

[7] J. Antoni, F. Bonnardot, A. Raad, M. El Badaoui, Mechanical Systems and Signal Processing 18 (2004) 1285-1314. Cyclostationary modelling of rotating machine vibration signals.

[8] K.R. Fyfe and D.S. Munck, Mechanical Systems and Signal Processing 11 (2) (1997) 187-205. Analysis of computed order tracking.

[9] P.D. McFadden, Mechanical Systems and Signal Processing 3 (1) (1989) 87-97. Interpolation techniques for time domain averaging of gear vibration. 
[10] F. Candocia, J.C. Principe, IEEE Transactions on Signal Processing 45 (7) (1998) 2044-2047. Comments on sinc interpolation of discrete periodic signals.

[11] T.J. Lim, M.D. Macleod, IEEE Signal Processing Letters 3 (5) May 1996, 144146. On-line interpolation using spline functions.

[12] R.B. Randall, J. Antoni, S. Chobsaard, Mechanical Systems and Signal Processing 15 (5) (2001) 945-962. The relationship between spectral correlation and envelope analysis in the diagnostics of bearing faults and other cyclostationary machine signals.

[13] B.D. Forrester, Advanced Vibration Analysis Techniques for Fault Detection and Diagnosis in Geared Transmission Systems, Ph.D dissertation (1996), Swinburne University of Technology, Melbourne, Australia. 